\title{
Approaching the Molecular Mechanism of Autophagy
}

\author{
Per E. Stromhaug and Daniel J. Klionsky* \\ Department of Biology, University of Michigan, Ann Arbor, \\ MI 48109, USA \\ ${ }^{*}$ Corresponding author: Daniel J. Klionsky, \\ klionsky@umich.edu
}

\begin{abstract}
Autophagy is a complex cellular process that involves dynamic membrane rearrangements under a range of physiological conditions. It is a highly regulated process that plays a role in cellular maintenance and development, and has been implicated in a number of genetic diseases. Upon induction of autophagy, cytoplasm is sequestered into vesicles and delivered to a degradative organelle, the vacuole in yeast or the lysosome in mammalian cells. The process is unique in that it converts material that is topologically intracellular into topologically extracellular. Autophagy was first described more than $\mathbf{5 0}$ years ago, but it is since the discovery of the pathway in yeast cells that our knowledge about the molecular events taking place during the process has expanded. The generation of autophagy-specific mutants in a variety of yeast cell lines has provided insight into functional roles of more than 15 novel genes, double that number if we include genes whose products function also in other processes. Although we have learned much about autophagy, many questions remain to be answered. This review highlights the most recent advances in the autophagy field in both yeast and mammalian cells.
\end{abstract}

Key words: autophagy, cytoplasm to vacuole targeting, degradation, lysosome, pexophagy, vacuole, yeast

Received 11 May 2000, revised and accepted for publication 24 May 2001

Cell growth and homeostasis is governed by tightly regulated biosynthetic and catabolic processes. In addition, cells need a certain degree of flexibility to adapt to changing environmental and/or developmental conditions. This adaptability may necessitate extensive turnover of cellular components including organelles, and eukaryotic cells harbor two powerful hydrolytic machineries for such purposes: the proteasome and the lysosome/vacuole. In both degradative systems, the hydrolytic arsenal is compartmentalized to avoid unregulated breakdown. In the case of the proteasome, only ubiquitintagged proteins are transported into the particle core where the proteolytic activity is hidden. The lysosome/vacuole, however, constitutes a hydrolase-packed organelle and imposes the need for mechanisms for delivering substrates destined for degradation across a delimiting membrane. Accordingly, eukaryotic cells have evolved a range of complex processes for transport of proteins and organelles from the cytoplasm to the lysosome/vacuole.

\section{Autophagy}

\section{Macroautophagy}

Autophagy was originally described as a cellular response to starvation. In mammalian cells deprived of serum and amino acids, vesicles containing bulk proteins and organelles rapidly appear within the cytosol and lysosome. These autophagosomes have a half-life in the range of minutes before lysosomal hydrolases start to degrade the macromolecular cargo, allowing the release of the monomeric units back into the cytosol. This form of autophagy is sometimes referred to as macroautophagy (Figure 1) and seems to take place in all eukaryotic cells, although the morphology may appear different from cell to cell. Yeast cells form double-membrane-bound autophagosomes with a diameter of $300-900 \mathrm{~nm}$, while some mammalian cells form multilamellar autophagosomes. Freeze-fracture studies of autophagosomes from rat hepatocytes and of yeast cells show that these vesicles are almost completely lacking transmembrane proteins $(1,2)$. The relative absence of membrane proteins fits with a role for the autophagosome as a carrier vesicle that is destined to be degraded by the hydrolytic arsenal of the lysosome/vacuole.

Starvation-induced autophagy is generally assumed to be nonselective with the purpose of generating building blocks needed by the cells to survive starvation. Autophagy allows haploid yeast cells to survive severe starvation for weeks; without autophagy, yeast cells start to die within 2 days under conditions of nutrient deprivation. Diploid yeast cells will not sporulate upon starvation if autophagy is inhibited. In mammalian organisms, organs with a high capacity for autophagy, such as the liver, may support the rest of the organism with macromolecular building blocks during starvation. Autophagy is probably the main mechanism for degradation of long-lived proteins and the only mechanism for turnover of organelles including mitochondria and peroxisomes. Although this turnover generally may occur randomly, degradation of peroxisomes by the related process of pexophagy may be highly selective. This sometimes selective property of autophagy has led to suggestions that autophagy may be an essential process during embryogenesis and cell differentiation 


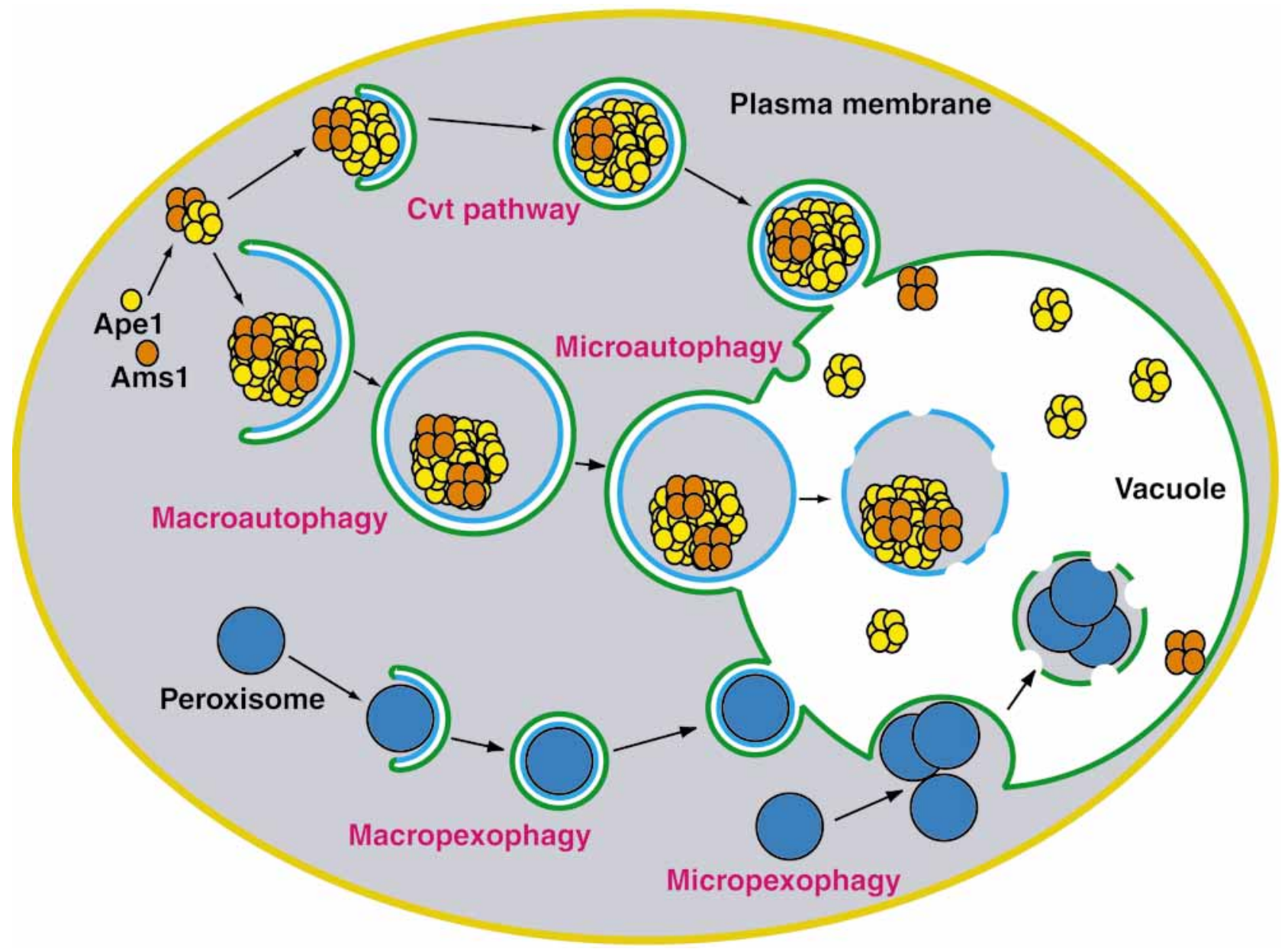

Figure 1: Morphology of autophagy, pexophagy and the Cvt pathway in yeast. Macroautophagy, macropexophagy and the Cvt pathway involve the formation of sequestering vesicles in the cytosol that engulf bulk cytoplasm, peroxisomes or prApe1 and Ams1, respectively. Following completion, these vesicles target to and fuse with the vacuole, releasing single-membrane vesicles within the lumen where they are degraded, allowing maturation of prApe1 and recycling of cytoplasm. In microautophagy and micropexophagy, cytoplasm and peroxisomes, respectively, are taken up directly at the vacuole surface.

\section{Pexophagy}

Selective autophagy of peroxisomes has been shown in both yeast and mammalian cells. After the cessation of treatment with peroxisome proliferators, the superfluous peroxisomes are degraded in a process that is inhibited by 3-methyladenine, a characteristic typical of autophagy. This degradation is also inhibited by long-chain fatty acids, substrates for peroxisomal $\beta$-oxidation. Most yeast cells are also capable of selective autophagy of peroxisomes. Saccharomyces cerevisiae grown on oleic acid, and Pichia pastoris and Hansenula polymorpha grown on methanol, will induce peroxisomal enzymes leading to a dramatic expansion of the peroxisomal compartment and/or the number of peroxisomes. If a different carbon source such as glucose is added, these peroxisomes are no longer required and are rapidly degraded within the vacuole. In $S$. cerevisiae and $H$. polymorpha the process used to take up the peroxisomes resembles starvation-induced macroautophagy, involving cytosolic seques- tration within autophagosome-like vesicles (Figure 1) $(3,4)$. This process is termed macropexophagy.

$P$. pastoris utilizes macropexophagy during ethanol adaptation and micropexophagy during glucose adaptation. Micropexophagy resembles a form of autophagy that has been seen to take place in vivo in mammalian cells as well as by purified mammalian lysosomes, and involves a smallscale invagination of the lysosomal membrane (Figure 1). During micropexophagy in $P$. pastoris, the whole vacuole engulfs the peroxisomes. This can be followed by electron and fluorescence microscopy, allowing pexophagy genes to be functionally classified as to whether they are involved in induction of pexophagy, recognition and sequestration of peroxisomes, homotypic fusion completing the uptake, and/or degradation (5). Although macropexophagy and micropexophagy of peroxisomes are morphologically distinct, they seem to require the same set of proteins as starvation-in- 
duced macroautophagy $(4,6)$. So far, only one protein has been described that is required for pexophagy but not for autophagy in P. pastoris and S. cerevisiae: Gsa9 and Cvt9, respectively (7). Interestingly, Cvt9 is also required for a constitutive, selective form of autophagy termed the cytoplasm-to-vacuole-targeting (Cvt) pathway in S. cerevisiae.

\section{The Cvt pathway}

Although the term autophagy reflects cellular self-cannibalism, this pathway may also serve a biosynthetic purpose in $S$. cerevisiae. The vacuole-resident hydrolase aminopeptidase I (Ape1) is not delivered to the vacuole by the ER and Golgi route, but instead is synthesized as a precursor (prApe1) in the cytosol, where it rapidly forms a dodecamer of $732 \mathrm{kDa}$ (Figure 1). This dodecamer forms a higher-order complex, and the delivery of this large complex to the vacuole requires the same set of proteins as starvation-induced autophagy and pexophagy $(4,6)$. The Cvt pathway also utilizes some proteins that are not required for nonspecific macroautophagy, such as Cvt9 (7). Another component specific to the Cvt pathway is Cvt19, a peripheral membrane protein shown in vivo and in vitro to have the characteristics of a prApe 1 receptor (8). Cvt19 interacts with the prApe1 propeptide that is known to contain vacuolar targeting information and is required for import of prApe1 under both vegetative and starvation conditions, but is not needed for nonspecific autophagy. In addition, cvt19s is the only characterized cvt or apg mutant that is not defective for pexophagy, suggesting that its role is restricted to biosynthetic import. Cvt19 is also required for delivery of a second resident hydrolase, $\alpha$-mannosidase (Ams1), to the vacuole (9). Similarly to prApe1, Ams 1 oligomerizes in the cytosol before sequestration, but it is not yet known whether they travel to the vacuole separately or in a shared vesicle.

A third protein that is required primarily for the Cvt pathway is Vac8, which is also involved in early vacuole migration during cell division. Vac8 interacts with Apg13 and may be part of a regulatory complex that controls the conversion between the Cvt and autophagy pathways (10) (see 'The Switching Complex' below). In addition to these proteins, the formation of Cvt vesicles also seems to require a different SNARE complex than the formation of autophagosomes (see 'Fusion and Degradation' below).

\section{Autophagy-related degradation pathways}

Several vacuolar/lysosomal degradation pathways besides autophagy have been described in mammalian and yeast cells, but it is not yet clear if they share any components with autophagy, except in the final degradation step. The best-described of these is chaperone-mediated autophagy, a process characterized in mammalian cells that involves the recognition of a degenerate KFERQ pentapeptide motif in proteins by the heat-shock cognate protein Hsc73, followed by Lamp2a-dependent translocation across the lysosomal membrane (reviewed in 11). A similar mechanism for vacuolar in vitro uptake of glyceraldehyde-3-phosphate dehydrogenase in S. cerevisiae requiring $\mathrm{Hsp} 70$ s has recently been described (12).
The activity of Hsp70 protein Ssa2 is also required for vacuolar degradation of fructose 1,6-bisphosphatase (FBPase) by the Vid pathway. FBPase is a key regulatory enzyme in the gluconeogenesis pathway, and is degraded upon addition of glucose. Vacuolar degradation of FBPase involves import of the enzyme into single membrane-bound Vid vesicles of $30 \mathrm{~nm}$ that subsequently fuse with the vacuole. The protein translocation requires Vid24, Ssa2 (13) and multiubiquitin chain formation by Ubc1 (14). A population of FBPase may also be degraded through the action of the proteasome.

A microautophagic pathway proposed to play a role in vacuolar membrane breakdown has recently also been described in $S$. cerevisiae (Figure 1). However, vacuolar uptake by this pathway is only partially blocked in autophagy mutants, indicating that this process may not be analogous to micropexophagy in $P$. pastoris $(15,16)$.

\section{Regulation of Autophagy}

The mechanism by which cells sense starvation and initiate a signal transduction event that induces autophagy is largely uncharacterized. In mammalian cells, regulatory factors may include protein kinases (such as Tor, and Erk1/2) and phosphatases (such as PP2A), G-proteins, calcium, and lipid kinases (reviewed in 17). In $S$. cerevisiae, the regulation involves a switch from the Cvt pathway, which operates under vegetative conditions, to autophagy. In P. pastoris, the onset of microautophagy of peroxisomes during glucose adaptation has been shown to require phosphofructokinase I (Pfk1) independent of the enzyme's glycolytic activity. Control of ethanol-induced macropexophagy probably occurs through an alternate mechanism that may involve glyoxylate (reviewed in 17). In agreement with the separate regulation of these two modes of pexophagy, pfk $1 /$ gsa 1 mutants are not defective for macropexophagy.

\section{Tor2}

The immunosuppressive drug rapamycin when complexed with FKBP inhibits Tor kinases in yeast and mammalian cells. By inhibiting Tor2, rapamycin mimics the effect of starvation, leading to a variety of cellular responses associated with $G_{0}$ arrest (reviewed in 18), including alterations in the phosphorylation patterns of autophagy-specific proteins, increased level of the autophagy protein Aut7, and the onset of nonselective autophagy.

\section{Trimeric G proteins}

Overexpressing the alpha-subunit of the heterotrimeric Gprotein $\mathrm{G}_{i 3}$ in HT-29 cells induces autophagy. The GDPbound form of the protein promotes autophagic uptake, while GTP-bound $\mathrm{G \alpha}_{\mathrm{i} 3}$ reduces autophagy. Hydrolysis of GTP on $\mathrm{G}_{\mathrm{i} 3}$ and stimulation of autophagy is accelerated by $\mathrm{G} \alpha$ interacting protein (GAIP). The activity of GAIP is further upregulated by phosphorylation by Erk1/2 MAP kinases, which in turn are phosphorylated in the absence of amino acids (19). The autophagy deficiency seen with a GTPase-deficient 
mutant of the $\mathrm{G}_{\mathrm{i} 3}$ protein may be partly overcome by overexpressing the Vps15 homolog p150 or synthetic phosphoinositide 3-phosphate, suggesting that $\mathrm{G} \alpha_{\mathrm{i} 3}$ acts upstream of the type III phosphoinositides.

\section{Phosphoinositide 3-kinase}

While type I phosphoinositide 3-phosphates are inhibitory for autophagy, type III are required (20). In mammalian cells, the PI 3-kinase inhibitor wortmannin is a potent inhibitor of autophagy, and it has also been shown that the selective autophagy inhibitor 3-methyladenine acts by inhibiting PI 3-kinase (21). Reduction of type III PI 3-kinase activity with antisense RNA greatly reduces autophagy in HT-29 cells (20). In S. cerevisiae, the PI 3-kinase Vps34 is a key enzyme in several transport events leading to the vacuole. Vps34, complexed with the protein kinase $V p s 15$ and the accessory proteins Vps38 and Vps30/Apg6, is essential for sorting of CPY to the vacuole, while a similar complex in which Vps38 is replaced with Apg 14 is essential for autophagy (22) (Figure 2).

A putative human homolog of Vps30/Apg6, the Bcl-2-interacting, coiled-coil protein Beclin 1, is able to complement only the autophagy deficiency in yeast cells lacking Vps30/ Apg6 (23). Beclin 1 is a candidate tumor suppressor, and reintroduction of Beclin 1 into the breast cancer cell line MCF7 not only restores autophagy in these cells but also suppresses the tumorigenicity and other malignant characteristics associated with cancer cells. In HeLa cells, all of Beclin 1 is associated with PI 3-kinase activity and most of this complex is localized to the trans-Golgi network (24). This localization of Beclin 1 could suggest a role for the phosphatidylinositol 3-phosphates in sorting of proteins to the autophagic organelle rather than as a regulatory effector. Morpho- logical studies in $H$. polymorpha and $P$. pastoris suggest that the activity of $\mathrm{Vps} 34 / \mathrm{Pdd} 1$ and $\mathrm{Vps} 15$ are required at an early stage of selective and nonselective autophagy $(3,25)$.

\section{Molecular Events in Autophagosome Formation}

Although we still do not know how autophagosomes form or all the factors required for this process, characterization of the known autophagy proteins has provided significant insight into how the proteins interact and function. Almost all of this knowledge comes from studies in yeast cells, but protein homologs exist in mammalian cells, and the characterization of these proteins and their involvement in autophagy has just begun. Two molecular pathways seem to play an important role in the initiation and sequestration process.

\section{The switching complex}

As discussed above, $S$. cerevisiae switches between the Cvt pathway and autophagy depending on nutrient conditions. A protein complex that may play a role in this regulatory switch has been identified $(7,10,26)$. A central component in this protein complex is Apg1, a serine/threonine protein kinase essential to both starvation-induced and selective autophagy (Figure 3). The substrate for this kinase is unknown, but using a synthetic substrate it has been shown that the kinase activity of Apg 1 is stimulated by starvation and rapamycin (26). Although the phosphorylation of Apg 1 itself is reduced upon starvation, activation of this protein appears to be controlled in part through binding to Apg13. Apg13 is hyperphosphorylated under rich conditions, while starvation induces dephosphorylation $(10,26)$. Apg13 also displays a higher affinity for
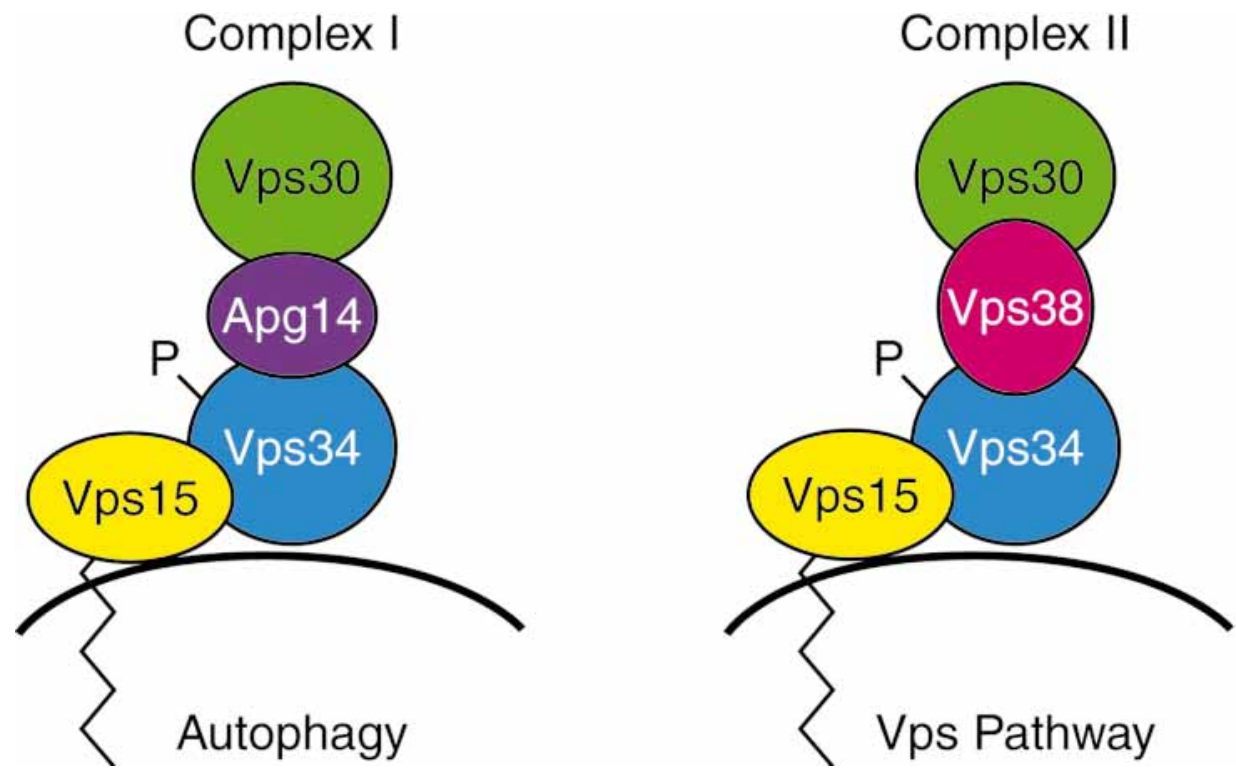

Figure 2: Two different PI 3-kinase complexes are involved in vacuolar protein targeting. Apg14 and Vps38 are specific components of a PI 3-kinase complex that is required for the autophagy/Cvt pathway or the Vps pathway, respectively. Vps30/Apg6, the Vps15 protein kinase and the Vps34 PI 3-kinase are common to both pathways. Adapted from Kihara et al., J Cell Biol 2001;152:519-530. 


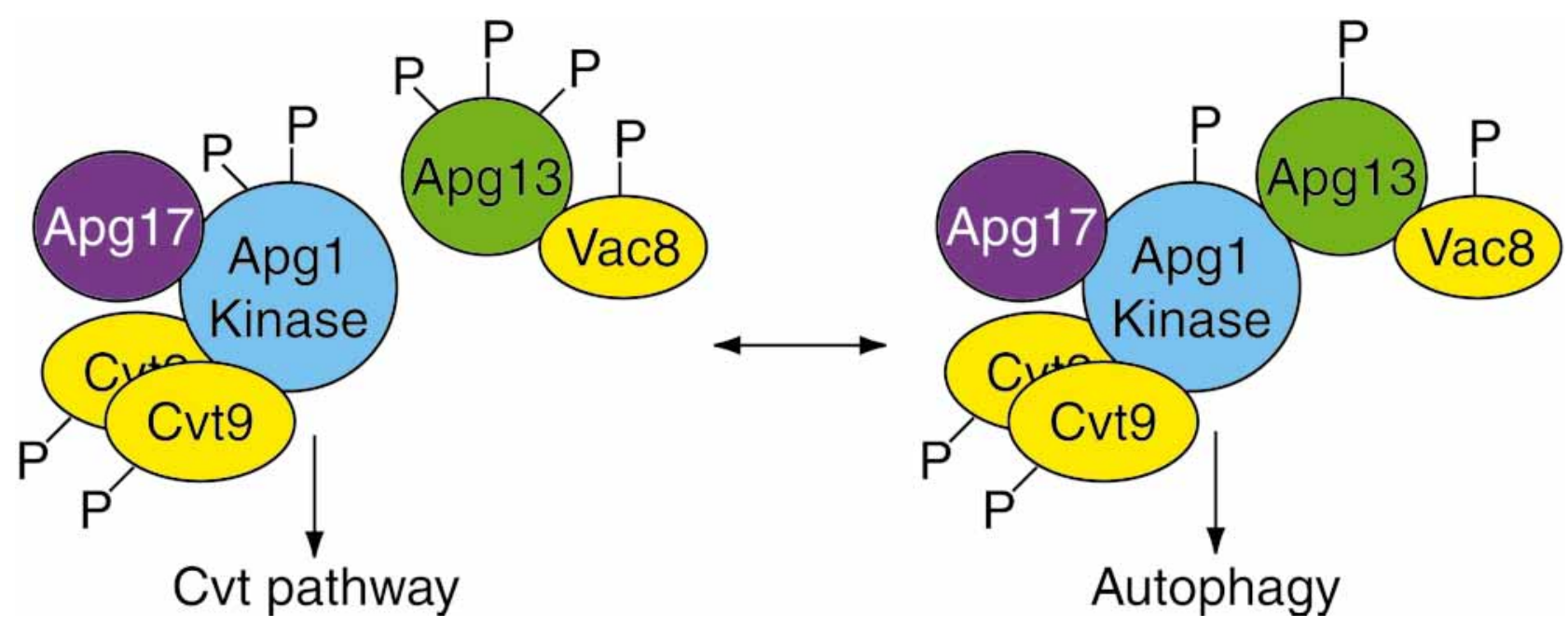

Figure 3: The Apg1 kinase complex includes components that are specific for the Cvt pathway or macroautophagy. The Apg1 kinase interacts with Cvt9 and Vac8, two components that are specific for the Cvt pathway, and Apg 17, a protein that is required only for autophagy. The interaction with Vac8 appears to be indirect and mediated through Apg13. The phosphorylation of at least Apg1 and Apg13 varies depending on the nutritional state of the cell. In starvation conditions or following treatment with rapamycin, both proteins are partially dephosphorylated and interact with a higher affinity. The phosphorylation state of Apg17 has not been determined.

Apg1 under starvation conditions. The tighter binding of Apg 13 to Apg 1 and subsequent activation of Apg1 occur concomitantly with a switch from selective cargo uptake to nonselective, bulk autophagy. Apg13 is further associated with Vac8, the vacuolar inheritance protein that also acts in the Cvt pathway but is not essential for starvation-induced autophagy (10). Vac8 is also a phosphoprotein and may help to facilitate phosphorylation of Apg 13 .

Apg1 also interacts with two other proteins. One of these, Apg 17, is not required for the Cvt pathway but has been proposed to play a function in the Apg1-Apg13 interaction (26). The other, Cvt9, is not required for non-selective autophagic import (7). Cvt9 is a large coiled-coil protein that localizes to a punctate structure close to the vacuole in $S$. cerevisiae and plays a role in both the Cvt pathway and pexophagy. In $P$. pastoris, the Cvt9 homolog Gsa9 also stains the vacuolar membrane. During micropexophagy, GFP-tagged Gsa9 seems to mediate tethering of the vacuolar membrane to the peroxisomes. In S. cerevisiae, Cvt9 stabilizes the binding of prAPI to the sequestering membrane. Cvt9 interacts with itself, possibly through the coiled-coil domain, and this could potentially crosslink the Apg1 complex into a higher-order structure required early in the sequestration process.

A putative homolog of Apg1 in C. elegans, Unc-51, is required for normal neuronal elongation. Ultrastructural analysis of the unc51 mutant revealed abnormal vesicles and membranous structures within the axons. A defect in axonal elongation has also been found by overexpressing a dominant negative mouse homolog in cultured cerebellar granule neurons (27). Two putative human homologs of Apg1 exist: ULK1 and ULK2. ULK1 has been shown by yeast two-hybrid screening to interact with GATE-16 and GABARAP, two homologs of the yeast autophagy protein Aut7 (see 'The Conjugation Cascade' below) (28).

\section{The conjugation cascade}

A hallmark in the autophagy field was the discovery that the two autophagy proteins Apg5 and Apg12 are physically connected through the action of Apg7 and Apg10 (29) (Figure 4). Apg7 and the P. pastoris homolog Gsa7 were identified as homologs of the ubiquitin-activating enzyme E1, with particularly strong homology around the ATP-binding domain $(6,30,31)$. Upon activation of an essential cysteine in Apg7 with ATP, the C-terminal glycine in Apg 12 is bound to Apg7 through a thioester linkage. The binding of Apg12 requires the $\mathrm{C}$-terminal 40 amino acids of Apg7 and facilitates dimerization of Apg7 (32). Apg12 is next transferred to a cysteine in Apg10, which acts as an E2 enzyme in conjugating Apg 12 through an isopeptide bond to internal lysine 149 in Apg5 (33). The small coiled-coil protein Apg16 binds preferentially to the conjugate and may crosslink conjugates through homo-oligomerization (34). Human homologs of Apg5 and Apg12 are also conjugated. In cultured mouse embryonic stem cells, Agp5 is required for autophagosome formation, and GFP-tagged human Apg5 can be used to trace the formation of autophagosomes from crescent-shaped cytoplasmic structures (35). Conjugation is not required for targeting of Apg 5 to the sequestering membrane but for elongation of the membrane into cupshaped structures. However, upon completion of the sequestration process, neither of the conjugation proteins remains associated with the nascent autophagosome.

A second conjugation event involving the membrane recruitment of Aut7 is also needed for autophagy, pexophagy and the Cvt pathway $(36,37)$ (Figure 4). Aut7 is a small protein without sequence similarity to ubiquitin but with a three-dimensional 


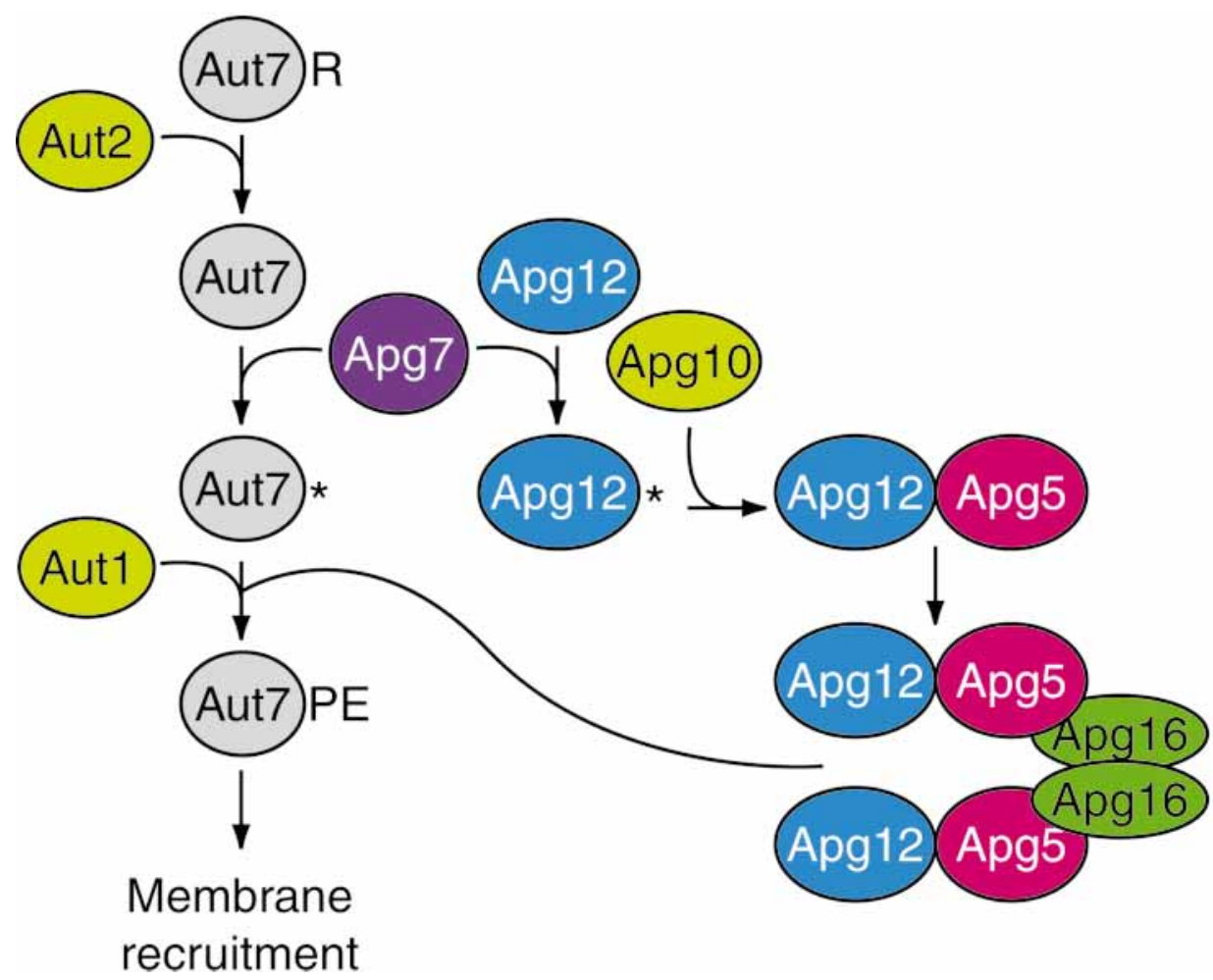

Figure 4: Membrane recruitment of Aut7 involves two separate conjugation reactions. Apg12 is activated by the E1-like enzyme Apg7 and conjugated to Apg5 through the action of Apg10. Apg16 dimerizes and links a pair of Apg12-Apg5 conjugates. Aut7 is processed by the Aut2 cysteine protease, activated by Apg7 and conjugated to phosphatidylethanolamine (PE) by Aut1. Attachment of PE and the action of the Apg12-Apg5 conjugation complex are required for membrane recruitment of Aut7.

structure predicted to contain an ubiquitin fold (38). Aut7 is synthesized with a C-terminal arginine that is immediately clipped off by Aut2, a cysteine protease, exposing a glycine $(39,40)$. Again, Apg7 is the activating enzyme that forms a thioester bond with the exposed glycine of Aut7. The Aut1 protein, acting like an E2 enzyme, then conjugates the protein to phosphatidylethanolamine (41). Aut7 is the only autophagy protein upregulated (10-20-fold) by starvation and displays a strong perivacuolar localization that is dependent on the Apg12-Apg5 conjugation pathway, as well as lipidation by Aut1. One of the primary distinguishing features between the Cvt pathway and autophagy is the size/capacity of the sequestering vesicles. If upregulation of Aut7 is inhibited during starvation by protein synthesis inhibitors, abnormally small autophagosomes are formed (42). A function for Aut7 in elongation of the sequestering membrane has therefore been proposed.

Three mammalian homologs, seemingly having very different functions, exist for Aut7: GABARAP, GATE-16 and MAP-LC3. GABARAP binds $\mathrm{GABA}_{A}$-receptor and promotes clustering of the receptor and binding to microtubules (43). GATE-16 was identified as a protein stimulating intra-Golgi transport in vitro and has been shown by immunofluorescence to reside in the Golgi complex (44). GATE-16 interacts with NSF and the Golgi v-SNARE GOS-28. S. cerevisiae Aut7 may substitute for GATE-16 in vitro, and interacts physically with the yeast
v-SNAREs Bet1 (ER to Golgi transport) and Nyv1 (vacuolar inheritance), and genetically with Sec22 (ER to Golgi), suggesting that Aut7 may exert several functions in yeast (45). Finally, MAP-LC3, is enriched as a processed form in autophagosomal membranes in HeLa cells (46). Removal of the last 22 amino acids as well as a second modification is required for autophagosome association (46) as well as conjugation of Apg12 to Apg5 (35). Interestingly, all three Aut7 homologs interact physically with the human homolog of Apg7 and may therefore be conjugated to proteins or lipids (47).

\section{Fusion and degradation}

The mechanism of formation/completion of the sequestering vesicle in macroautophagy and the Cvt pathway is far from understood. However, this process is likely to involve components in a SNARE complex, either during the addition of new membrane for vesicle expansion, or in a final homotypic fusion event that completes the vesicle. The syntaxin and Sec1 homologs Tlg2 and Vps45, respectively, are required for import of prApe1 but not autophagy (48). This finding suggests that different SNARE components are required for autophagy and the CVt pathway, and possibly that different membranes are used to form the sequestering vesicles. Following completion of the double-membrane vesicle in the cytosol, the outer membrane fuses with the vacuole/lysosome, releasing 
the inner single-membrane vesicle into the lumen. A distinct docking step has not been shown to occur in autophagic import but is likely to take place, based on the overlap of the fusion machinery with that of other vacuolar import pathways, as well as homotypic vacuole fusion (reviewed in 17). Ypt7, Vam3, Vti1 and the components of the class C/HOPS complex are required for all characterized fusion events involving the vacuole.

After release into the lumen, the subvacuolar vesicle is degraded to allow access to the cargo. Degradation must be regulated to prevent breakdown of the outer vesicle membrane that is now continuous with the vacuole/lysosome membrane. Degradation requires a low $\mathrm{pH}$, proteinase $\mathrm{B}$ (Prb1) and Cvt17. Prb1 is a hydrolase that is involved in the activation of many other vacuolar zymogens. Thus, Prb1 may play an indirect role in vesicle breakdown. At present, Cvt17 is the only putative lipase that has been identified as having a role in subvacuolar vesicle degradation and has homology with triacylglycerol lipases (49). The mechanism or site of action of Cvt17 is unknown, as the protein was not reported to be present at high levels in the vacuole.

\section{Concluding Remarks}

Autophagy is a common cellular process that is involved in a wide range of physiological events. It is an essential process in microorganisms under stress conditions such as starvation, a situation that is frequently encountered in the natural environment. In multicellular organisms, autophagy is a homeostatic mechanism that may represent the first stage in a cellular/tissue response that can range from autophagy to apoptosis and necrosis (50). In this sense, autophagy is a protective mechanism that can eliminate cells that would otherwise prove harmful to the organism. In fact, autophagy has been suggested to play a critical role in type II (nonapoptotic) programmed cell death (reviewed in 50). Defects in autophagy have been linked to numerous diseases in mammals, including cancer, cardiomyopathy and neurodegenerative defects. Continued analysis of autophagy and the related processes of cytoplasm to vacuole targeting and pexophagy in both yeasts and mammalian cells will provide an exciting avenue for future work in this important area of cell biology.

\section{Acknowledgments}

The authors apologize to those researchers whose work has not been included or cited due to space limitations. This work is supported by National Institutes of Health Public Health Service Grant GM53396 to D.J.K. and by a grant from The Research Council of Norway to P.E.S.

\section{References}

1. Fengsrud M, Erichsen ES, Berg TO, Raiborg C, Seglen PO. Ultrastructural characterization of the delimiting membranes of isolated auto- phagosomes and amphisomes by freeze-fracture electron microscopy. Eur J Cell Biol 2000;79:871-882.

2. Baba M, Osumi M, Ohsumi Y. Analysis of the membrane structures involved in autophagy in yeast by freeze-replica method. Cell Struct Funct 1995;20:465-471.

3. Kiel JAKW, Rechinger KB, Van der Klei IJ, Salomons FA, Titorenko VI, Veenhuis M. The Hansenula polymorpha PDD1 gene product, essential for the selective degradation of peroxisomes, is a homologue of Saccharomyces cerevisiae Vps34p. Yeast 1999;15:741-754.

4. Hutchins MU, Veenhuis M, Klionsky DJ. Peroxisome degradation in Saccharomyces cerevisiae is dependent on machinery of macroautophagy and the Cvt pathway. J Cell Sci 1999;112:4079-4087.

5. Sakai Y, Koller A, Rangell LK, Keller GA, Subramani S. Peroxisome degradation by microautophagy in Pichia pastoris. Identification of specific steps and morphological intermediates. J Cell Biol 1998; $141: 625-636$.

6. Yuan W, Stromhaug PE, Dunn WA Jr. Glucose-induced autophagy of peroxisomes in Pichia pastoris requires a unique E1-like protein. Mol Biol Cell 1999;10:1353-1366.

7. Kim J, Kamada Y, Stromhaug PE, Guan J, Hefner-Gravink A, Baba M, Scott SV, Ohsumi Y, Dunn WA Jr, Klionsky DJ. Cvt9/Gsa9 functions in sequestering selective cytosolic cargo destined for the vacuole. J Cell Biol 2001;153:381-396.

8. Scott SV, Guan J, Hutchins MU, Kim J, Klionsky DJ. Cvt19 is a receptor for the cytoplasm to vacuole targeting pathway. Mol Cell 2001 ; in press.

9. Hutchins MU, Klionsky DJ. Vacuolar localization of oligomeric $\alpha$-mannosidase requires the cytoplasm to vacuole targeting and autophagy pathway components in Saccharomyces cerevisiae. J Biol Chem $2001 ; 276: 20491-20498$.

10. Scott S, Nice D III, Nau J, Weisman L, Kamada Y, Keizer-Gunnink I, Funakoshi T, Veenhuis M, Ohsumi Y, Klionsky DJ. Apg13p and Vac8p are part of a complex of phosphoproteins that are required for cytoplasm to vacuole targeting. J Biol Chem 2000;275:25840-25849.

11. Cuervo AM, Dice JF. Lysosomes, a meeting point of proteins, chaperones, and proteases. J Mol Med 1998;76:6-12.

12. Horst M, Knecht EC, Schu PV. Import into and degradation of cytosolic proteins by isolated yeast vacuoles. Mol Biol Cell 1999;10:28792889.

13. Brown CR, McCann JA, Chiang HL. The heat shock protein Ssa2p is required for import of fructose-1, 6-bisphosphatase into Vid vesicles. J Cell Biol 2000;150:65-76.

14. Shieh HL, Chen Y, Brown CR, Chiang HL. Biochemical analysis of fructose-1,6-bisphosphatase import into vacuole import and degradation vesicles reveals a role for $U B C 1$ in vesicle biogenesis. J Biol Chem $2001 ; 276: 10398-10406$.

15. Sattler T, Mayer A. Cell-free reconstitution of microautophagic vacuole invagination and vesicle formation. J Cell Biol 2000;151:529-538.

16. Muller O, Sattler T, Flotenmeyer M, Schwarz H, Plattner H, Mayer A. Autophagic tubes. vacuolar invaginations involved in lateral membrane sorting and inverse vesicle budding. J Cell Biol 2000;151:519528.

17. Kim J, Klionsky DJ. Autophagy, cytoplasm-to-vacuole targeting pathway, and pexophagy in yeast and mammalian cells. Annu Rev Biochem 2000;69:303-342.

18. Schmelzle T, Hall MN. TOR, a central controller of cell growth. Cell 2000;103:253-262.

19. Ogier-Denis E, Pattingre S, El Benna J, Codogno P. Erk1/2-dependent phosphorylation of $\mathrm{G} \alpha$-interacting protein stimulates its GTPase accelerating activity and autophagy in human colon cancer cells. J Biol Chem 2000;275:39090-39095.

20. Petiot A, Ogier-Denis E, Blommaart EF, Meijer AJ, Codogno P. Distinct classes of phosphatidylinositol $3^{\prime}$-kinases are involved in signaling pathways that control macroautophagy in HT-29 cells. J Biol Chem 2000;275:992-998. 
21. Blommaart EFC, Krause U, Schellens JPM. Vreeling-Sindelárová H Meijer AJ. The phosphatidylinositol 3-kinase inhibitors wortmannin and LY294002 inhibit autophagy in isolated rat hepatocytes. Eur J Biochem 1997:243:240-246.

22. Kihara A, Noda T, Ishihara N, Ohsumi Y. Two distinct Vps34 phosphatidylinositol 3-kinase complexes function in autophagy and carboxypeptidase $Y$ sorting in Saccharomyces cerevisiae. J Cell Biol $2001 ; 152: 519-530$.

23. Liang XH, Jackson S, Seaman M, Brown K, Kempkes B, Hibshoosh $\mathrm{H}$, Levine B. Induction of autophagy and inhibition of tumorigenesis by beclin 1. Nature 1999;402:672-676.

24. Kihara A, Kabeya Y, Ohsumi Y, Yoshimori T. Beclin-phosphatidylinositol 3-kinase complex functions at the trans-Golgi network. EMBO Rep $2001 ; 2: 330-335$.

25. Stasyk OV, van der Klei IJ, Bellu AR, Shen S, Kiel JA, Cregg JM, Veenhuis MA. Pichia pastoris VPS15 homologue is required in selective peroxisome autophagy. Curr Genet 1999;36:262-269.

26. Kamada Y, Funakoshi T, Shintani T, Nagano K, Ohsumi M, Ohsumi Y. Tor-mediated induction of autophagy via an Apg1 protein kinase complex. J Cell Biol 2000;150:1507-1513.

27. Tomoda T, Bhatt RS, Kuroyanagi H, Shirasawa T, Hatten ME. A mouse serine/threonine kinase homologous to $C$. elegans UNC51 functions in parallel fiber formation of cerebellar granule neurons. Neuron 1999:24:833-846

28. Okazaki N, Yan J, Yuasa S, Ueno T, Kominami E, Masuho Y, Koga H, Muramatsu M. Interaction of the Unc-51-like kinase and microtubuleassociated protein light chain 3 related proteins in the brain: possible role of vesicular transport in axonal elongation. Brain Res Mol Brain Res 2000;85:1-12.

29. Mizushima N, Noda T, Yoshimori T, Tanaka Y, Ishii T, George MD, Klionsky DJ, Ohsumi M, Ohsumi Y. A protein conjugation system essential for autophagy. Nature 1998;395:395-398

30. Tanida I, Mizushima N, Kiyooka M, Ohsumi M, Ueno T, Ohsumi Y Kominami E. Apg7p/Cvt2p: a novel protein-activating enzyme essential for autophagy. Mol Biol Cell 1999;10:1367-1379.

31. Kim J, Dalton VM, Eggerton KP, Scott SV, Klionsky DJ. Apg7p/Cvt2p is required for the cytoplasm-to-vacuole targeting, macroautophagy, and peroxisome degradation pathways. Mol Biol Cell 1999;10:1337-1351.

32. Komatsu M, Tanida I, Ueno T, Ohsumi M, Ohsumi Y, Kominami E. The $\mathrm{C}$-terminal region of an Apg7p/Cvt2p is required for homodimerization and is essential for its E1 activity and E1-E2 complex formation. J Biol Chem 2001;276:9846-9854

33. Shintani T, Mizushima N, Ogawa Y, Matsuura A, Noda T, Ohsumi Y. Apg10p, a novel protein-conjugating enzyme essential for autophagy in yeast. EMBO J 1999;18:5234-5241.

34. Mizushima N, Noda T, Ohsumi Y. Apg16p is required for the function of the Apg12p-Apg5p conjugate in the yeast autophagy pathway. EMBO J 1999;18:3888-3896.

35. Mizushima N, Yamamoto A, Hatano M, Kobayashi Y, Kabeya Y, Suzuki K, Tokuhisa T, Ohsumi Y, Yoshimori T. Dissection of autophagosome formation using Apg5-deficient mouse embryonic stem cells. J Cell Biol 2001:152:657-667.
36. Kirisako T, Baba M, Ishihara N, Miyazawa K, Ohsumi M, Yoshimori T, Noda T, Ohsumi Y. Formation process of autophagosome is traced with Apg8/Aut7p in yeast. J Cell Biol 1999;147:435-446.

37. Huang W-P, Scott SV, Kim J, Klionsky DJ. The itinerary of a vesicle component, Aut7p/Cvt5p, terminates in the yeast vacuole via the autophagy/Cvt pathways. J Biol Chem 2000;275:5845-5851.

38. Paz Y, Elazar Z, Fass D. Structure of GATE-16, membrane transport modulator and mammalian ortholog of autophagocytosis factor Aut7p. J Biol Chem 2000;275:25445-25450.

39. Kirisako T, Ichimura $Y$, Okada H, Kabeya $Y$, Mizushima N, Yoshimori T, Ohsumi M, Takao T, Noda T, Ohsumi Y. The reversible modification regulates the membrane-binding state of Apg8/Aut7 essential for autophagy and the cytoplasm to vacuole targeting pathway. J Cell Biol 2000;151:263-276

40. Kim J, Huang W-P, Klionsky DJ. Membrane recruitment of Aut7p in the autophagy and cytoplasm to vacuole targeting pathways requires Aut $1 p$, Aut2p, and the autophagy conjugation complex. J Cell Biol $2001 ; 152: 51-64$.

41. Ichimura $Y$, Kirisako T, Takao T, Satomi $Y$, Shimonishi $Y$, Ishihara N, Mizushima N, Tanida I, Kominami E, Ohsumi M, Noda T, Ohsumi Y. A ubiquitin-like system mediates protein lipidation. Nature 2000;408: 488-492.

42. Abeliovich H, Dunn WA Jr, Kim J, Klionsky DJ. Dissection of autophagosome biogenesis into distinct nucleation and expansion steps. J Cell Biol 2000;151:1025-1034

43. Chen L, Wang HB, Vicini S, Olsen RW. The $\gamma$-aminobutyric acid type $A\left(G A B A_{A}\right)$ receptor-associated protein (GABARAP) promotes $G_{A B} A_{A}$ receptor clustering and modulates the channel kinetics. Proc Natl Acad Sci USA 2000;97:11557-11562

44. Sagiv $Y$, Legesse-Miller A, Porat A, Elazar Z. GATE-16, a membrane transport modulator, interacts with NSF and the Golgi v-SNARE GOS 28. EMBO J 2000;19:1494-1504

45. Legesse-Miller A, Sagiv Y, Glozman R, Elazar Z. Aut7p, a soluble autophagic factor, participates in multiple membrane trafficking processes. J Biol Chem 2000;275:32966-32973.

46. Kabeya $Y$, Mizushima N, Ueno T, Yamamoto A, Kirisako T, Noda T, Kominami E, Ohsumi Y, Yoshimori T. LC3, a mammalian homologue of yeast Apg8p, is localized in autophagosome membranes after processing. EMBO J 2000;19:5720-5728.

47. Tanida I, Tanida-Miyake E, Ueno T, Kominami E. The human homolog of Saccharomyces cerevisiae Apg7p is a protein-activating enzyme for multiple substrates including human Apg12p, GATE-16, GABARAP, and MAP-LC3. J Biol Chem 2001:276:1701-1706.

48. Abeliovich H, Darsow T, Emr SD. Cytoplasm to vacuole trafficking of aminopeptidase I requires a t-SNARE-Sec1p complex composed of Tlg2p and Vps45p. EMBO J 1999;18:6005-6016.

49. Teter SA, Eggerton KP, Scott SV, Kim J, Fischer AM, Klionsky DJ Degradation of lipid vesicles in the yeast vacuole requires function of Cvt17, a putative lipase. J Biol Chem 2001;276:20832087.

50. Klionsky DJ, Emr SD. Autophagy as a regulated pathway of cellular degradation. Science 2000:290:1717-1721. 